\title{
Determination of polarized parton distribution functions and their uncertainties
}

\author{
M. Hirai,, , : S. Kumano, ${ }^{2, \text { t }}$ and N. Saito ${ }^{3, \text { }}$ \\ (Asymmetry Analysis Collaboration) \\ ${ }^{1}$ Radiation Laboratory, RIKEN (The Institute of Physical and Chemical Research) \\ Wako, Saitama 351-0198, JAPAN \\ ${ }^{2}$ Department of Physics, Saga University, Saga, 840-8502, Japan \\ ${ }^{3}$ Department of Physics, Kyoto University, Kyoto, 606-8502, Japan
}

(Dated: November 7, 2018)

\begin{abstract}
We investigate the polarized parton distribution functions (PDFs) and their uncertainties by using the world data on the spin asymmetry $A_{1}$. The uncertainties of the polarized PDFs are estimated by the Hessian method. The up and down valence-quark distributions are determined well. However, the antiquark distributions have large uncertainties at this stage, and it is particularly difficult to fix the gluon distribution. The $\chi^{2}$ analysis produces a positively polarized gluon distribution, but even $\Delta g(x)=0$ could be allowed according to our uncertainty estimation. In comparison with the previous AAC (Asymmetry Analysis Collaboration) parameterization in 2000, accurate SLAC-E155 proton data are added to the analysis. We find that the E155 data improve the determination of the polarized PDFs, especially the polarized antiquark distributions. In addition, the gluon-distribution uncertainties are reduced due to the correlation with the antiquark distributions. We also show the global analysis results with the condition $\Delta g(x)=0$ at the initial scale, $Q^{2}=1 \mathrm{GeV}^{2}$, for clarifying the error correlation effects with the gluon distribution.
\end{abstract}

PACS numbers: 13.60.Hb,13.88.+e

\section{INTRODUCTION}

The spin structure of the nucleon has been investigated extensively since the discovery of the EMC spin effect [1]. Despite a naive expectation that the nucleon spin is carried by quarks, the experimental result indicated that only a small fraction is carried by the quarks. In order to determine this quark spin content and internal spin structure, the determination of the first moments of the polarized parton distribution functions (PDFs) is necessary. Furthermore, the $x$ and $Q^{2}$ dependence of these functions is crucial in the calculation of the polarized cross sections. The functions should be determined from global analyses of polarized experimental data. Such analyses have been made by several groups 2, 3, 4, 5, 6, 7, 8, 9, 10, 11, 12, 13]. Now, there are available data for the spin asymmetry $A_{1}$ or the structure function $g_{1}$ by polarized deep inelastic scattering (DIS) experiments [1, 14, 15, 16, 17]. These data are valuable especially for fixing the the polarized valence quark distributions $\Delta u_{v}(x)$ and $\Delta d_{v}(x)$. The polarized antiquark distributions are still not well determined from the data. In particular, their flavor dependence is not shown reliably at this stage. Furthermore, the polarized gluon distribution cannot be fixed from the polarized DIS data although there are some constraints.

The above statements describe the current status of

\footnotetext{
*E-mail: mhirai@rarfaxp.riken.jp

${ }^{\dagger}$ E-mail: kumanos@cc.saga-u.ac.jp

${ }^{\ddagger}$ E-mail: saito@nh.scphys.kyoto-u.ac.jp
}

global analyses qualitatively well; however, we have been longing for more quantitative discussion on the uncertainties in the PDFs. Recently, uncertainty estimation methods have been developed for the unpolarized PDFs. Mathematical formulations of PDF uncertainties are proposed in Refs. 18, 19]. Practical methods are also developed [20, 21, 22, 23] and they are actually used in recent unpolarized analyses [24, 25, 26]. Thanks to a large number of experimental data points with excellent precision and wide kinematical coverage, the unpolarized PDFs are well determined from small $x$ to large $x$ with a reasonable precision so that hadronic cross sections can be calculated to a few percent accuracy [24, 26, 27]. In the same way, the uncertainties of the polarized PDFs have been investigated [12, 13]. The polarized gluon distribution has large uncertainties due to a lack of data which are sensitive to the distribution. However, because polarized experimental projects are going on, we expect to have better determination of $\Delta g$ in the near future. The determination of the polarized PDF uncertainties enables the uncertainty estimation of physical observables such as scattering cross sections and spin asymmetries. The uncertainty estimation of the polarized PDFs is valuable for understanding the present situation and for clarifying the importance of future experiments.

There are three major purposes in this paper. First, the uncertainties of the polarized PDFs are investigated for the AAC (Asymmetry Analysis Collaboration) parameterization 6]. Although there are uncertainty estimations for the polarized PDFs 12, 13], the results could depend on the parameterized functional form and the details of the uncertainty estimation method. Therefore, it 
is important to estimate the PDF uncertainties independently. In particular, we discuss the large uncertainties of the polarized gluon distribution. Second, we investigate the impact of precise SLAC-E155 proton data, which are not included in the previous AAC analysis, on the PDF uncertainties, especially on those of the polarized antiquark and gluon distributions. Third, error correlation is investigated by a global analysis with $\Delta g(x)=0$ at the initial $Q^{2}$ point. We compare its PDF uncertainties with those of the $\Delta g(x) \neq 0$ analysis in order to show error correlation effects.

This paper is organized as follows. In Sec. III we describe the framework of our parameterization for the polarized PDFs. The Hessian method is explained in Sec. III as an uncertainty estimation method for the PDFs. In Sec. IV $\chi^{2}$ analysis results are shown with the polarized PDF uncertainties. First, they are compared with the experimental data for the spin asymmetry $A_{1}$. Second, obtained polarized PDFs are shown in comparison with the distributions of the previous AAC version and other parameterization studies. Third, effects of the SLAC-E155 data are explained, and the correlation between the antiquark and gluon distribution uncertainties is discussed. The results are summarized in Sec. D

\section{PARAMETERIZATION OF POLARIZED PARTON DISTRIBUTION FUNCTIONS}

The major source of information on the polarized PDFs has been polarized electron and muon DIS experiments. The polarized PDFs are determined by comparing theoretical functions with the asymmetry $A_{1}$ data of the polarized DIS experiments [1, 14, 15, 16, 17]. The variable $Q^{2}$ is given by $Q^{2}=-q^{2}$ with the momentum transfer $q$, and the scaling variable $x$ is defined by $x=Q^{2} /(2 p \cdot q)$ with the nucleon momentum $p$. We selected the data with $Q^{2}>1 \mathrm{GeV}^{2}$ so that perturbative QCD could be applied relatively safely. Then, the total number of available data is 399, and they cover the kinematical region, $0.004<x<0.75$ and $1<Q^{2}<72$ $\mathrm{GeV}^{2}$.

The spin asymmetry $A_{1}$ is expressed in terms of the polarized structure function $g_{1}$, the unpolarized structure function $F_{2}$, and the longitudinal-transverse structure function ratio $R$ :

$$
A_{1}\left(x, Q^{2}\right)=\frac{g_{1}\left(x, Q^{2}\right)}{F_{2}\left(x, Q^{2}\right)} 2 x\left[1+R\left(x, Q^{2}\right)\right] .
$$

The SLAC analysis results 28] are used for the function $R\left(x, Q^{2}\right)$. The structure function $F_{2}\left(x, Q^{2}\right)$ is expressed by unpolarized PDFs:

$$
\begin{aligned}
F_{2}\left(x, Q^{2}\right)=\sum_{i=1}^{n_{f}} e_{i}^{2} x & \left\{C_{q}\left(x, \alpha_{s}\right) \otimes\left[q_{i}\left(x, Q^{2}\right)+\bar{q}_{i}\left(x, Q^{2}\right)\right]\right. \\
& \left.+C_{g}\left(x, \alpha_{s}\right) \otimes g\left(x, Q^{2}\right)\right\}
\end{aligned}
$$

Here, $q\left(x, Q^{2}\right), \bar{q}\left(x, Q^{2}\right)$, and $g\left(x, Q^{2}\right)$ are the quark, antiquark, and gluon distributions, respectively, and $C_{q}\left(x, \alpha_{s}\right)$ and $C_{g}\left(x, \alpha_{s}\right)$ are coefficient functions. The symbol $\otimes$ denotes the convolution integral:

$$
f(x) \otimes g(x)=\int_{x}^{1} \frac{d y}{y} f\left(\frac{x}{y}\right) g(y) .
$$

In the same way, the structure function $g_{1}\left(x, Q^{2}\right)$ is expressed as

$$
\begin{array}{r}
g_{1}\left(x, Q^{2}\right)=\frac{1}{2} \sum_{i=1}^{n_{f}} e_{i}^{2}\left\{\Delta C _ { q } ( x , \alpha _ { s } ) \otimes \left[\Delta q_{i}\left(x, Q^{2}\right)\right.\right. \\
\left.\left.+\Delta \bar{q}_{i}\left(x, Q^{2}\right)\right]+\Delta C_{g}\left(x, \alpha_{s}\right) \otimes \Delta g\left(x, Q^{2}\right)\right\}
\end{array}
$$

where $\Delta q\left(x, Q^{2}\right), \Delta \bar{q}\left(x, Q^{2}\right)$, and $\Delta g\left(x, Q^{2}\right)$ are the polarized quark, antiquark, and gluon distributions, respectively. The function $\Delta q$ is defined by $\Delta q=q^{\uparrow}-q^{\downarrow}$, which indicates the difference between the distribution of quark with helicity parallel to that of parent nucleon and the one with helicity anti-parallel. The functions $\Delta C_{q}\left(x, \alpha_{s}\right)$ and $\Delta C_{g}\left(x, \alpha_{s}\right)$ are the polarized coefficient functions.

As the polarized PDF at the initial scale $Q_{0}^{2}$, we choose the functional form:

$$
\Delta f(x)=\left[\delta x^{\nu}-\kappa\left(x^{\nu}-x^{\mu}\right)\right] f(x),
$$

where $f(x)$ is the corresponding unpolarized PDF. This form is taken for imposing the positivity condition and for reducing correlations among the parameters. Optimized PDFs are four distributions: $\Delta u_{v}(x), \Delta d_{v}(x), \Delta \bar{q}(x)$, and $\Delta g(x)$, which are defined at $Q_{0}^{2}$ by Eq. (5). The $\delta$, $\nu, \kappa$, and $\mu$ are free parameters, which are determined by a $\chi^{2}$ analysis.

In principle, the separation of these quark distributions can be arbitrarily chosen. For example, alternative choice would be $\Delta u^{+}(x), \Delta d^{+}(x)$ and $\Delta s^{+}(x)$, where

$$
\Delta u^{+}(x)=\Delta u(x)+\Delta \bar{u}(x),
$$

and similar expressions for $\Delta d^{+}(x)$ and $\Delta s^{+}(x)$. Here, these quark distributions can be related to ours as

$$
\begin{aligned}
\Delta u^{+}(x) & =\Delta u_{v}(x)+2 \Delta \bar{q}(x) \\
\Delta d^{+}(x) & =\Delta d_{v}(x)+2 \Delta \bar{q}(x) \\
\Delta s^{+}(x) & =2 \Delta \bar{q}(x)
\end{aligned}
$$

Here $\Delta \bar{q}(x)$ can simply be interpreted as an average of polarized sea-quark distributions.

Practical difference appears, however, when we apply constraints on the first moments of the quark distributions from the axial coupling constants of octet baryons. By denoting the first moments by $\int_{0}^{1} \Delta f(x) d x=\Delta F$, these moments should be connected to

$$
\begin{aligned}
\Delta U^{+}-\Delta D^{+} & =1.267 \pm 0.011 \\
\Delta U^{+}+\Delta D^{+}-2 \Delta S^{+} & =0.585 \pm 0.025 .
\end{aligned}
$$


These relations can be rewritten by using our separation of quark distributions,

$$
\begin{aligned}
& \Delta U_{v}-\Delta D_{v}=1.267 \pm 0.011-2 \Delta_{2}, \\
& \Delta U_{v}+\Delta D_{v}=0.585 \pm 0.025-4 \Delta_{3} .
\end{aligned}
$$

where

$$
\begin{aligned}
& \Delta_{2} \equiv \Delta \bar{U}-\Delta \bar{D}, \\
& \Delta_{3} \equiv \frac{\Delta \bar{U}+\Delta \bar{D}}{2}-\Delta \bar{S} .
\end{aligned}
$$

Since there is no experimental guidance on the size of the first moments of the flavor asymmetric distributions $\left(\Delta_{2}\right.$ and $\Delta_{3}$ ), we continue to neglect them in this paper as was done in our previous one, although we are also preparing a new calculation with those breaking parameters. This point will be discussed later in this section.

The polarized distributions are numerically evolved to the $Q^{2}$ points of experimental data by the DGLAP (Dokshitzer-Gribov-Lipatov-Altarelli-Parisi) evolution equations [29] in order to calculate $\chi^{2}$. The total $\chi^{2}$ is defined by

$$
\chi^{2}=\sum_{i} \frac{\left[A_{1, i}^{\text {data }}\left(x, Q^{2}\right)-A_{1, i}^{\text {calc }}\left(x, Q^{2}\right)\right]^{2}}{\left[\Delta A_{1, i}^{\text {data }}\left(x, Q^{2}\right)\right]^{2}},
$$

where $\Delta A_{1}^{\text {data }}$ is the experimental error including both systematic and statistical errors: $\left(\Delta A_{1}^{\text {data }}\right)^{2}=$ $\left(\Delta A_{1}^{\text {stat }}\right)^{2}+\left(\Delta A_{1}^{\text {syst }}\right)^{2}$. The total $\chi^{2}$ is minimized by the CERN subroutine MINUIT [30].

Here, the systematic errors $\Delta A_{1}^{\text {syst }}$ are fully included. It would be ideal to include uncorrelated and correlated systematic errors separately so that we can perform fully consistent uncertainty analysis. The issue of the correlated errors in the global analysis is indeed investigated in the recent unpolarized PDF parametrizations 24, 25]. In the polarized PDF analysis, however, these errors are not listed separately in papers and it is very difficult to access such information. Because of this incompleteness, our analyses overestimate the uncertainty in the PDFs.

In order to obtain a rough picture of the effects of systematic uncertainties, we also performed $\chi^{2}$ calculation with the statistical uncertainties only, which resulted in $\sim 20 \%$ increase in the $\chi^{2}$. In average, this increase in $\chi^{2}$ corresponds to the fact that quadratic sum of statistical error and full systematic error is larger by $\sim 10 \%$. By looking at the individual data points, the increase in the errors ranges from a few percent to $50 \%$, which is not inconsistent with the average picture. From this exercise, we can conclude that our uncertainties of the PDFs are overestimated, but only by $\sim 10 \%$ in average, although we now emphasize the needs of separated systematic errors so that we can perform fully consistent uncertainty analyses in future.

In comparison with the previous AAC analysis [6], the SLAC-E155 proton data are added to the $\chi^{2}$ analysis. In order to demonstrate the impact of this new data set, we used the same configuration with the previous analysis. The renormalization scheme is the $\overline{\mathrm{MS}}$ scheme in the next-to-leading order (NLO). The initial scale is set at $Q_{0}^{2}=1 \mathrm{GeV}^{2}$. The number of flavor is fixed at $N_{f}=3$ and heavy flavor contributions are neglected.

We use the GRV98 NLO parameter set as the unpolarized PDFs 31]. Even if other unpolarized PDFs, for example CTEQ6 [24], are used, the results do not change significantly. In particular, the polarized gluon distribution is modified; however, it is well within the uncertainties for $\Delta g(x)$ in Sec. IVB We should also mention that unpolarized PDF uncertainties are not included in our analysis for estimating the polarized PDF uncertainties. The value of $\Lambda_{Q C D}$ is taken from the GRV unpolarized PDF analysis: $\Lambda_{Q C D}^{N_{f}=3}=299 \mathrm{MeV}$. Unpolarized experimental data are generally more accurate than polarized ones, so that $\Lambda_{Q C D}$ had better be determined by the unpolarized analysis.

In the following, we discuss two important constraints, the positivity and flavor-symmetric conditions, on the polarized PDF determination.

\section{A. The positivity condition}

The positivity condition means that the magnitude of a polarized cross section should be smaller than the corresponding unpolarized one: $|\Delta \sigma| \leq \sigma$. In the leading order (LO), this relation indicates $|\Delta f(x)| \leq f(x)$ because probabilistic interpretation can be applied for the parton distributions. However, the condition is not strictly satisfied in the NLO because of higher-order corrections 32]. The correction due to the coefficient functions is small in the limit $x \rightarrow 1$. Therefore, the positivity condition $|\Delta f(x)| \leq f(x)$ could be used practically for constraining the polarized PDFs at large $x$.

If this condition is not taken into account in the $\chi^{2}$ analysis, it tends to be violated significantly in the polarized antiquark and gluon distributions: $[|\Delta f(x)| / f(x)]_{x \rightarrow 1} \gg 1$. It could lead to an unphysical cross section: $|\Delta \sigma|>\sigma$. This unfavorable behavior comes from the lack of accurate experimental data in the large- $x$ region. Furthermore, experimental data indicate that the spin asymmetry $A_{1}$ increases monotonically as a function of $x$ in the large- $x$ region. It easily leads to unphysical results without the positivity constraint. Therefore, the positivity condition for the polarized PDFs is practically a useful constraint for avoiding the unphysical results, and we decided to impose this condition in the $\chi^{2}$ analysis. The condition restricts the range of the parameter $\delta$ which controls the large- $x$ behavior of the polarized PDFs: $-1 \leq \delta \leq 1$.

\section{B. Flavor symmetric antiquark distributions}

It is now known that unpolarized antiquark distributions, $\bar{u}, \bar{d}$, and $\bar{s}$, are different from lepton scattering 
and Drell-Yan experiments [33]. There are model explanations, for example, by meson clouds, chiral soliton, and Pauli exclusion. These models are extended to the polarized antiquark distributions. There are available experimental data which may indicate the polarized flavor dependence [8, 34, 35]; however, they are not accurate enough to find the difference between $\Delta \bar{u}$ and $\Delta \bar{d}$. Therefore, there is no reliable data for fixing the difference between the polarized antiquark distributions, and the determination of the flavor asymmetric distributions still depend on separation models [8, 9, 12].

Even in the alternative quark separation, $\Delta u^{+}(x)$, $\Delta d^{+}(x)$ and $\Delta s^{+}(x)$, we cannot address these specific questions. In future, the flavor dependence of the polarized antiquark distributions will be investigated, for example, at RHIC by $W$ production [36]. If these data become available, it makes sense to introduce the flavor dependent parameters into the $\chi^{2}$ analysis.

With the flavor symmetric assumption, i.e. $\Delta_{2}=$ $\Delta_{3}=0$, the first moments of $\Delta u_{v}$ and $\Delta d_{v}$ are fixed from Eq. (9): $\Delta U_{v}=0.926$ and $\Delta D_{v}=-0.341$.

\section{PDF UNCERTAINTY}

The uncertainties of the parton distributions are estimated by the Hessian method, which has been used as a general statistical method for estimating errors. The uncertainties come from measurement errors in the global PDF analysis.

The parameters, e.g. $\delta, \nu, \kappa$, and $\mu$ for each distribution in our analysis, are denoted $a_{i}(i=1,2, \cdots, N)$, where $N$ is the number of optimized parameters. Expanding $\chi^{2}$ around the minimum point $\hat{a}$ and keeping the leading quadratic terms, we have

$$
\Delta \chi^{2} \equiv \chi^{2}(\hat{a}+\delta a)-\chi^{2}(\hat{a})=\sum_{i, j} H_{i j} \delta a_{i} \delta a_{j}
$$

where the Hessian $H_{i j}$ is the second derivative matrix in the function $\chi^{2}(a)$. The first derivative terms do not exist because they vanish at the minimum point.

For estimating the PDF uncertainty, we should evaluate a confidence region of the multivariate normal distribution for optimized-parameter errors. Equation (12) indicates the local behavior around $\hat{a}$, and the confidence region could be identified by an ellipsoid which is defined by $\Delta \chi^{2}$. Assuming parabolic curves for the function $\chi^{2}(a)$, we can vary the ellipsoid to an arbitrary confidence level by choosing $\Delta \chi^{2}$. In our estimation, the $\Delta \chi^{2}$ value is obtained by the following procedure.

The confidence level $P$ for the normal distribution with $N$ degrees of freedom is identified with the one for the $\chi^{2}$ distribution:

$$
P=\int_{0}^{\Delta \chi^{2}} \frac{1}{2 \Gamma\left(\frac{N}{2}\right)}\left(\frac{S}{2}\right)^{\frac{N}{2}-1} \exp \left(-\frac{S}{2}\right) d S,
$$

where $\Gamma(m)$ is the Gamma function. It can be chosen to be the probability of one- $\sigma$-error range of the normal distribution $(P=0.6826)$ for our study in order to compare with the experimental errors. In the case of one parameter $(N=1)$, we obviously have $\Delta \chi^{2}=1$ from Eq. (13). Therefore, $\Delta \chi^{2}=1$ could be simply used for calculating the uncertainty if the parameter number is one. However, the parton distributions are provided with many parameters, so that the $\Delta \chi^{2}$ value should be re-evaluated. For example, the parameter number is eleven $(N=11)$ in our new polarized PDF analysis, and it leads to $\Delta \chi^{2}=12.647$.

The uncertainty of a parton distribution $F(x, \hat{a})$ with respect to the optimized parameters $\hat{a}$ is then calculated by using the Hessian matrices and assuming linear error propagation:

$$
[\delta F(x)]^{2}=\Delta \chi^{2} \sum_{i, j}\left(\frac{\partial F(x, \hat{a})}{\partial a_{i}}\right) H_{i j}^{-1}\left(\frac{\partial F(x, \hat{a})}{\partial a_{j}}\right) .
$$

For the PDF uncertainty estimation, we can analytically calculate the gradient terms $\partial F(x, \hat{a}) / \partial a_{i}$ at the initial scale $Q_{0}^{2}$. For the estimation at arbitrary $Q^{2}$, each gradient term is evolved by the DGLAP evolution kernel, and then the PDF uncertainties $\delta \Delta f\left(x, Q^{2}\right)$ are calculated. The uncertainties of the polarized structure functions $g_{1}^{p}$, $g_{1}^{n}$, and $g_{1}^{d}$ are calculated by the convolution integrals of the PDF gradient terms with the coefficient functions.

\section{RESULTS AND DISCUSSION}

We report our analysis results. Because one of the major purposes is to show the PDF uncertainties, we analyzed the data only for the NLO set. In our new analysis, the best fit is obtained with $\chi^{2}(/$ d.o.f. $)=346.5(0.893)$.

TABLE I: Numbers of the $A_{1}$ data with $Q^{2}>1 \mathrm{GeV}^{2}$ and $\chi^{2}$ values are listed. The notations $p, n$, and $d$ indicate proton, neutron, and deuteron, respectively.

\begin{tabular}{ccc}
\hline \hline data set & No. of data & $\chi^{2}$ \\
\hline EMC $(p)$ & 10 & 4.5 \\
SMC $(p)$ & 59 & 54.0 \\
E130 $(p)$ & 8 & 4.9 \\
E143 $(p)$ & 81 & 61.1 \\
$\operatorname{E155}(p)$ & 24 & 24.2 \\
HERMES $(p)$ & 19 & 19.1 \\
\hline SMC $(d)$ & 65 & 56.5 \\
E143 $(d)$ & 81 & 93.6 \\
E155 $(d)$ & 24 & 20.3 \\
\hline E142 $(n)$ & 8 & 2.6 \\
E154 $(n)$ & 11 & 3.6 \\
HERMES $(n)$ & 9 & 2.2 \\
\hline total & 399 & 346.5 \\
\hline \hline
\end{tabular}


TABLE II: Obtained parameters by the NLO analysis (AAC03).

\begin{tabular}{ccccc}
\hline \hline distribution & $\delta$ & $\nu$ & $\kappa$ & $\mu$ \\
\hline$\Delta u_{v}$ & $0.975 \pm 0.099$ & 0.000 (fixed) & 0.601 & $1.095 \pm 0.266$ \\
$\Delta d_{v}$ & $-1.000 \pm 0.377$ & 0.000 (fixed) & -0.721 & $1.318 \pm 0.466$ \\
$\Delta \bar{q}$ & $1.000 \pm 0.994$ & $1.014 \pm 0.182$ & $-90.96 \pm 13.57$ & 1.000 (fixed) \\
$\Delta g$ & $-1.000 \pm 3.959$ & $2.248 \pm 1.089$ & $254.2 \pm 180.7$ & $2.217 \pm 2.172$ \\
\hline \hline
\end{tabular}

The $\chi^{2}$ contributions from all the used data sets are listed in Table \and optimized parameters are summarized in Table III

In the previous AAC version (AAC00) [6], we found that an antiquark parameter $\mu_{\bar{q}}$, which determines the functional behavior of $\Delta \bar{q}$ at small $x$, cannot be fixed from the data because of the lack of small- $x$ data. Therefore, we fixed the parameter at $\mu_{\bar{q}}=1$ in our new analysis. Other four parameters are also fixed. The parameter $\nu_{u_{v}}$ and $\nu_{d_{v}}$ tended to stop at the positivity limit, so that these parameters are finally fixed. The parameters $\kappa_{u_{v}}$ and $\kappa_{d_{v}}$ are determined by the first moments $\Delta u_{v}$ and $\Delta d_{v}$ from semileptonic data with the assumption for the flavor symmetric antiquark distributions. The difference from the AAC00 NLO-2 analysis is the addition of the SLAC-E155 data. In order to discuss the influence of such accurate experimental data on the polarized PDF analysis, new analysis results are compared to the AAC00-NLO-2 in following subsections. The total number of the optimized parameters is eleven, so that the uncertainty is estimated by $\Delta \chi^{2}=12.647$ as explained in Sec. III

\section{A. Spin asymmetries}

We discuss the results for the spin asymmetries and their uncertainties. In addition to the data used for the AAC00 analysis, the SLAC-E155 proton target data are included. The E155 data cover the region, $0.015<x<$ 0.75 and $1.22<Q^{2}<34.72 \mathrm{GeV}^{2}$. Calculated spin asymmetries are shown in Fig. 11 and they are compared with the previous results (AAC00 NLO-2). The solid curves and shaded areas show the spin asymmetries $A_{1}^{p}, A_{1}^{n}$, and $A_{1}^{d}$ and their uncertainties of the new results (AAC03), respectively. The dashed and dasheddot curves indicate those of the AAC00 NLO-2. The $A_{1}$ uncertainties are calculated by using the estimated uncertainties for the obtained polarized PDFs.

The spin asymmetries are slightly modified especially in the region $0.02<x<0.3$. It is noteworthy that the E155 proton data also affect the spin asymmetries of the neutron and deuteron since $\Delta u_{v}(x)$ and $\Delta \bar{q}(x)$ are modified. Although the asymmetry modifications are rather small, the uncertainties are significantly modified. Comparing the shaded areas with the dashed-dot curves, we find that the addition of the E155 data reduces the $A_{1}$ uncertainties. The uncertainties in the region $x<0.6$
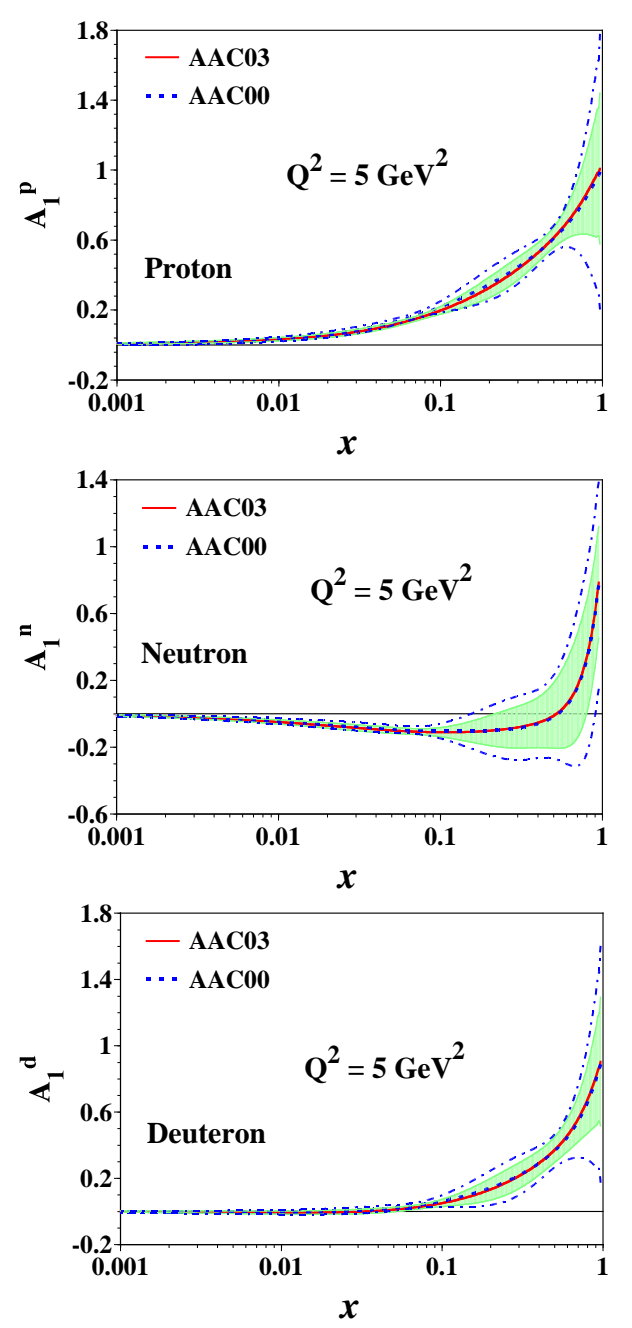

FIG. 1: Calculated spin asymmetries and their uncertainties are shown at $Q^{2}=5 \mathrm{GeV}^{2}$. The solid curves and shaded areas indicate the spin asymmetries and their uncertainties of the new results (AAC03), respectively. The dashed and dashed-dot curves show those of the previous results (AAC00 NLO-2).

are reduced directly by the E155 data. In addition, the data indirectly contribute to the uncertainty reduction in the large- $x$ region, where precise data are not available, through the $x$-dependent PDF form.

Next, the differences between the $A_{1}$ data and the theoretical asymmetries, namely $A_{1}^{\text {data }}-A_{1}^{A A C 03}$, are shown in Fig. 2] The shaded areas indicate the uncertainties of 

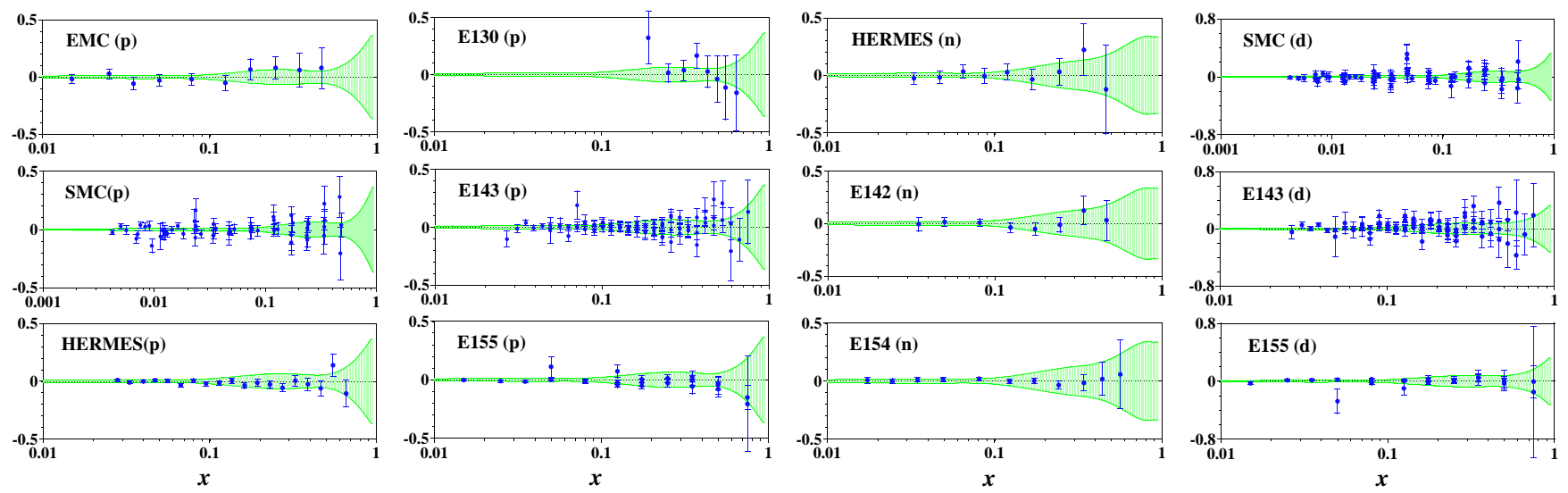

FIG. 2: Comparison of the AAC03 spin asymmetries with experimental data. The ordinates indicate the differences between experimental data and theoretical values $\left(A_{1}^{\text {data }}-A_{1}^{A A C 03}\right)$. The error bars indicate the errors obtained by the quadratic summations of the statistical and systematic errors. The shaded areas show the uncertainties at $Q^{2}=5 \mathrm{GeV}^{2}$.

the AAC03 analysis. The error bars indicate the ones obtained by the quadratic summations of the statistical and systematic errors. We find that the uncertainties are roughly equal to the errors of the experimental data. The spin asymmetries are constrained in the region $x<0.1$; however, they still have rather large uncertainties in the larger- $x$ region. It is obvious that the large- $x$ asymmetries are not determined well from the present data. These uncertainties at large $x$ imply that the positivity of the spin asymmetry is not necessarily guaranteed in the $\chi^{2}$ analysis unless the positivity condition is enforced. The shaded areas spread out in the region $0.1<x<0.6$ due to large errors of the E143 and SMC data for the proton. The numbers of these data are larger than those of HERMES and E155 experiments, so that their overall $\chi^{2}$ contributions are larger and the accurate E155 data cannot contribute much in this region. The situation is similar for the deuteron uncertainties. The neutron uncertainties are still large because the used ${ }^{3} \mathrm{He}$ target data are not accurate enough in comparison with the proton and deuteron data. We expect that the neutron uncertainties could be improved by precise JLab measurements [37].

\section{B. Polarized parton distribution functions}

We discuss obtained polarized PDFs and their uncertainties. In Fig. 3 the polarized PDFs of the AAC03 analysis are compared with those of the with the AAC00 NLO-2 at $Q^{2}=1 \mathrm{GeV}^{2}$. The distributions $\Delta u_{v}, \Delta \bar{q}$, and $\Delta g$ are slightly modified, but $\Delta d_{v}$ is scarcely changed. This is because $\Delta u_{v}$ is the dominant component of the proton structure function $g_{1}^{p}$ and because the whole seaquark contribution is in general larger than the $\Delta d_{v}$ contribution. Therefore, the larger components $\Delta u_{v}$ and $\Delta \bar{q}$ are mainly affected by the added precise E155 data.

Next, PDF uncertainties are shown at $Q^{2}=1 \mathrm{GeV}^{2}$ in Fig. 4. The shaded areas are the uncertainties of the
AAC03 analysis, and the dashed curves indicate those for the AAC00. The valence-quark distributions are sufficiently constrained by the polarized DIS data. However, we find rather large uncertainties in the region $0.1<x<0.6$, which corresponds to the region of the large $A_{1}$ uncertainties in Fig. 2 It indicates the necessity of accurate data in this kinematical region for better determination of the polarized valence-quark distributions. In particular, accurate ${ }^{3} \mathrm{He}$ data are useful for reducing the $\Delta d_{v}$ uncertainties because the $\Delta d_{v}$ contribution to $g_{1}^{n}$ is almost the same as the $\Delta u_{v}\left(g_{1}^{n} \propto 4 \Delta d_{v}+\Delta u_{v}+12 \Delta \bar{q}\right)$, whereas the contribution is small in $g_{1}^{p}$. On the other hand, the uncertainties of the antiquark and gluon distributions are still large. The huge gluon uncertainties indicate that the present data cannot rule out the possibility of $\Delta g(x)=0$ and negative gluon polarization, although the obtained gluon distribution is positive.
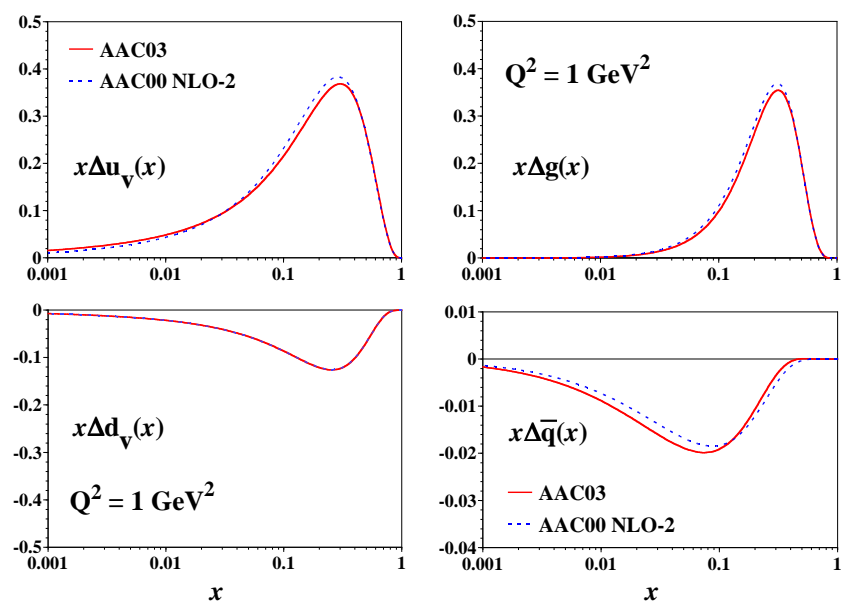

FIG. 3: Obtained polarized parton distributions at $Q^{2}=$ $1 \mathrm{GeV}^{2}$. The solid curves indicate the new AAC03 results, and the dashed curves are taken from the previous analysis (AAC00 NLO-2). 

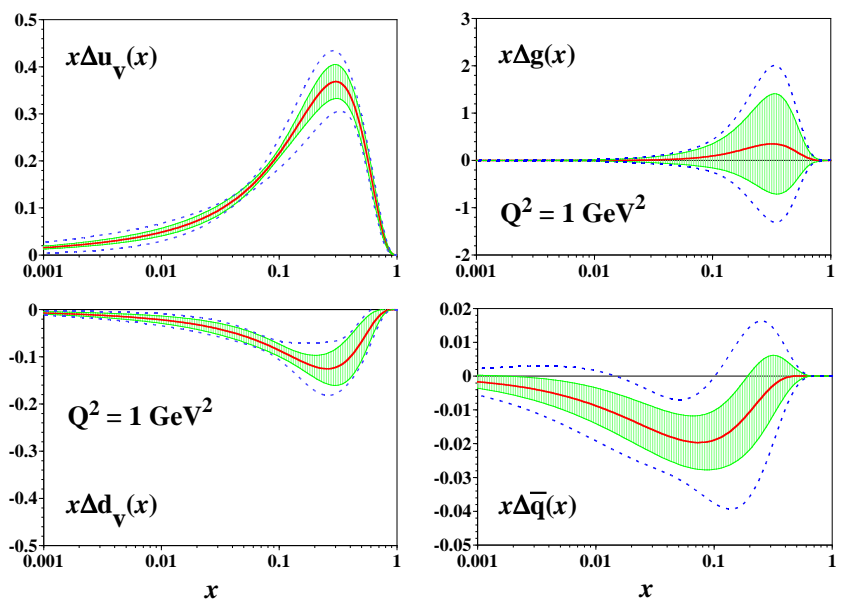

FIG. 4: Polarized PDF uncertainties are shown at $Q^{2}=1$ $\mathrm{GeV}^{2}$. The solid curves and shaded areas are the polarized PDFs and their uncertainties of the new AAC03 results, and the dashed curves are the uncertainties of the AAC00 results.

As shown in Fig. 4 all the PDF uncertainties are significantly reduced in the AAC03 analysis in comparison with the AAC00 because the accurate E155 data are added to the data set. In particular, the $\Delta d_{v}$ uncertainties are reduced although the $\Delta d_{v}$ distribution stays almost the same. In addition, the uncertainties of the antiquark and gluon distributions are significantly improved. The antiquark uncertainty reduction is directly due to the E155 data. However, it is difficult to understand that the significant reduction of the gluon uncertainties is due to the added new data. This is because that the gluon distribution indirectly contributes as a higher order correction with the coefficient function, and this contribution is less than quark contributions. The huge gluon uncertainties explicitly indicate the difficulty of fixing the gluon distribution from the DIS experimental data.

We find that the gluon uncertainty reduction is caused by an error correlation. The non-diagonal part of the Hessian indicates a strong correlation between the polarized antiquark and gluon distributions. The correlation affects the determination of these distributions. We discuss the details of the uncertainty improvement due to the correlation in Sec. IVE

\section{Quark spin content}

We show the first moments of the AAC03 parameterization at $Q^{2}=1 \mathrm{GeV}^{2}$ in Table III and they are compared with those of the AAC00 NLO-2 set. The first moments of the up- and down-valence quark distributions are fixed in both analyses. The first moments indicate that quarks carry about $20 \%$ of the parent nucleon spin, and gluons carry a large positive fraction of the nucleon spin. Their uncertainties are significantly reduced by the added E155 data; however, the present data are not enough to obtain accurate values, especially for the
TABLE III: The first moments of the obtained polarized PDFs at $Q^{2}=1 \mathrm{GeV}^{2}$. The AAC03 analysis results are compared to those of the previous results (AAC00 NLO-2). The $\Delta \Sigma$ is the quark spin content.

\begin{tabular}{cccc}
\hline \hline & $\Delta \bar{q}$ & $\Delta g$ & $\Delta \Sigma$ \\
\hline AAC03 & $-0.062 \pm 0.023$ & $0.499 \pm 1.266$ & $0.213 \pm 0.138$ \\
AAC00 & $-0.057 \pm 0.037$ & $0.533 \pm 1.931$ & $0.241 \pm 0.225$ \\
\hline \hline
\end{tabular}

gluon first moment.

The uncertainty of the spin content $\Delta \Sigma$ strongly depends on the antiquark uncertainty because it is given by $\Delta \Sigma_{N_{f}=3}=\Delta u_{v}+\Delta d_{v}+6 \Delta \bar{q}$. The first moments of the valence-quark distributions are fixed, so that the $\Delta \Sigma$ uncertainty is equal to six times the $\Delta \bar{q}$ uncertainty, which could be large due to the uncertainty of the distribution $\Delta \bar{q}(x)$ in the small- $x$ region. It suggests that the extrapolation into the smaller- $x$ region should be ambiguous in calculating the integral over $x$. We expect that accurate polarized antiquark distributions will be measured in future, then the quark spin content issue will become clear.

\section{Comparison with other parameterizations}

The AAC03 analysis results are compared with other parameterizations at $Q^{2}=1 \mathrm{GeV}^{2}$ in Fig. 5] For comparison, we choose three sets of the polarized PDFs in the NLO: GRSV01 (standard scenario) 9], BB (ISET=3) [13], and LSS ( $\overline{\mathrm{MS}}$ scheme) 12]. These parameterizations used basically the same experimental data set of the polarized DIS, but they choose averaged data tables over $x$ and $Q^{2}$, whereas full tables are used in the AAC analysis.
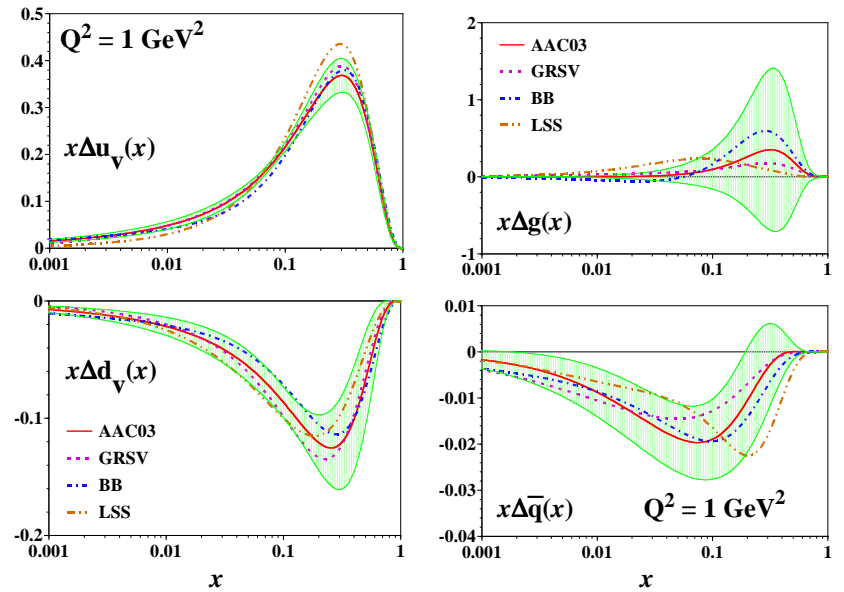

FIG. 5: The AAC03 PDFs at $Q^{2}=1 \mathrm{GeV}^{2}$ are compared with the ones for other parameterizations by GRSV01 (standard scenario) 9], BB (ISET=3) [13], and LSS ( $\overline{\mathrm{MS}}$ scheme) [12]. The shaded areas are the uncertainties of the AAC03 analysis. 
The flavor symmetric antiquark distributions are used in all these parameterizations.

Because the first moments are fixed in the same way, the variations are small in the polarized valence-quark distributions among the parameterizations in Fig. 5 There are slight variations in the antiquark distributions, and the gluon distributions differ significantly among the analysis groups. However, we find that all the parameterizations are consistent each other because the distributions are mostly within the estimated error bands.

The BB and LSS groups also investigated the polarized PDF uncertainties by the Hessian method. However, these uncertainties may not be directly compared with our uncertainties because the used $\Delta \chi^{2}$ value is not specified in their papers. In addition, uncertainty estimation methods are slightly different; for example, only the statistical errors are used and a relative normalization shift is introduced in the BB analysis. In general, the error estimations involve complicated systematic errors, e.g. functional form, data selection, and highertwist effects, in the global analyses, and they may not be estimated numerically. It is difficult to clarify these issues only by the current polarized DIS experiment data. Therefore, we need to investigate such hidden uncertainties when we improve the quality of the polarized PDFs by incorporating future experimental data.

\section{E. $\Delta g(x)=0$ analysis and error correlation}

In order to understand the reduction of the gluon uncertainty in Fig. [4 we discuss the error correlation between the polarized antiquark and gluon distributions. As the most simple assumption which could be allowed within the gluon uncertainties in Fig. 4 we choose
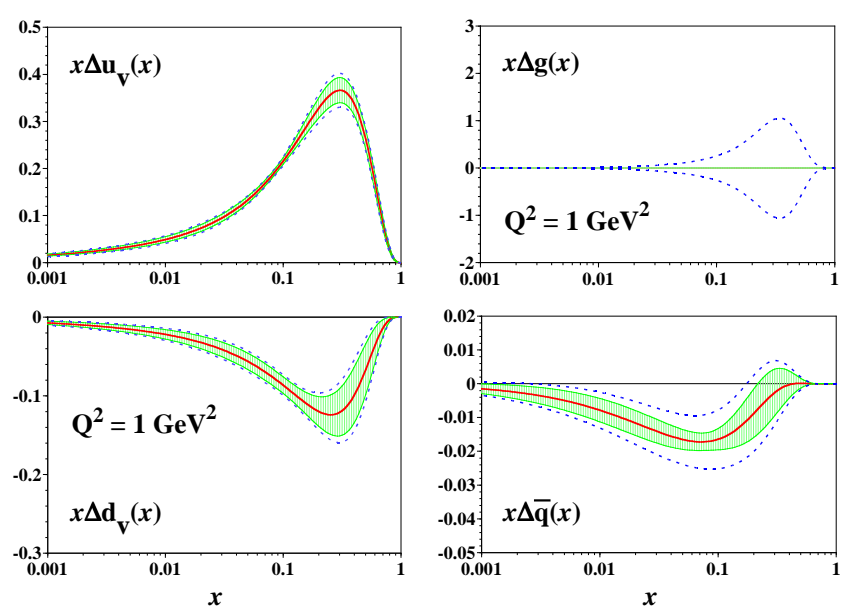

FIG. 6: The PDF uncertainties of the $\Delta g(x)=0$ analysis are compared with those of the $\Delta g(x) \neq 0$ analysis (AAC03). The solid curves indicate the polarized PDFs of the $\Delta g(x)=$ 0 analysis at $Q^{2}=1 \mathrm{GeV}^{2}$, and the shade areas are their uncertainties. The dashed curves indicate the uncertainties of the $\Delta g(x) \neq 0$ analysis.
$\Delta g(x)=0$ at the initial scale $\left(Q^{2}=1 \mathrm{GeV}^{2}\right)$. However, one should note that a finite distribution $\Delta g(x) \neq 0$ appears at larger $Q^{2}$ from the singlet $Q^{2}$ evolution. Since the gluon-distribution parameters are fixed, we can carry out an uncorrelated analysis with the gluon distribution.

In the $\Delta g(x)=0$ analysis, we obtain $\chi^{2}(/$ d.o.f. $)=$ $355.0(0.915)$, which is $2.5 \%$ larger than the value for the $\Delta g(x) \neq 0$ analysis. Because it is a slight change in the $\chi^{2}$ value, it is reasonable that $\Delta g(x)=0$ is allowed in Fig. 4 if the uncertainties are taken into account. Obtained polarized PDFs are shown in Fig. [6] for the $\Delta g(x)=0$ analysis. The total number of optimized parameters is seven for this analysis, so that the uncertainties are estimated by $\Delta \chi^{2}=8.180$. The calculated uncertainties are shown by the shaded areas, and they are compared with those of the $\Delta g(x) \neq 0$ analysis (AAC03) shown by the dashed curves. We find that the antiquark uncertainties are significantly reduced. On the other hand, the valence quark uncertainties are scarcely changed, which indicates that the correlation with the gluon distribution is weak even in the $\Delta g(x) \neq 0$ analysis.

It suggests that the antiquark distributions should be determined well by the present data if their errors are uncorrelated with those of the gluon. However, because of the existence of the strong error correlation, the actual antiquark uncertainties are increased by the huge gluon uncertainties. In future, if the polarized gluon distribution is measured accurately, the antiquark uncertainties also become small due to the strong correlation. To the contrary, the gluon uncertainties could be reduced by accurate measurements of the antiquark distributions. In this way, we find the significant reduction of the antiquark uncertainties in Fig. [6 is caused by the error correlation effects with the polarized gluon distribution. Furthermore, it indicates that the gluon uncertainty reduction in Fig. 4 is also due to the correlation effect between these distributions.

From these studies, it becomes clear that accurate determination of the gluon distribution is important also for the determination of the antiquark distributions. At this stage, the polarized gluon distribution is not accurately determined, and it also makes it difficult to fix the antiquark distributions from the DIS experimental data. In this sense, it is important to measure the polarized gluon distribution, for example, by direct photon production and jet production at RHIC.

\section{SUMMARY}

We have investigated the optimum polarized parton distributions by analyzing the polarized DIS data. We focused our studies particularly on three aspects, the uncertainty estimation of the obtained PDFs, the role of the accurate E155 proton data, and the error correlation between the polarized gluon and quark distributions.

First, the obtained PDF uncertainties indicated that the polarized valence-quark distributions are determined 
well, that the uncertainties of the polarized antiquark distributions are slightly large, and that the gluon uncertainties are huge. It is obvious that the polarized gluon distribution cannot be determined from the present DIS data.

Second, we discussed the role of accurate E155 data in the global analysis. Comparing the AAC00 and AAC03 results, we clarified that the E155 data contributed to reducing the $\mathrm{PDF}$ uncertainties significantly.

Third, the error correlation is investigated by repeating the parametrization analysis with the initial condition $\Delta g(x)=0$. In the $\Delta g(x)=0$ analysis, there is no error correlation between the gluon and quark distributions, and it leads to small uncertainties of the obtained antiquark distributions. This fact suggests that precise gluon measurements should be valuable for a better determination of the polarized antiquark distributions. The opposite way is also right: Precise polarized quark mea- surements should provide constraints for the gluon distribution.

Finally, we mention that the AAC03 polarized PDF library is available at the web site 38 . The polarized PDFs can be calculated numerically at given $x$ and $Q^{2}$ values.

\section{Acknowledgments}

The authors thank the AAC members, especially Y. Goto, H. Kobayashi, M. Miyama, and T.-A. Shibata, for discussions. S.K. was supported by the Grant-in-Aid for Scientific Research from the Japanese Ministry of Education, Culture, Sports, Science, and Technology. This work is partially supported by the Japan-U.S. Cooperative Science Program.
[1] European Muon Collaboration (EMC), J. Ashman et al., Phys. Lett. B206, 364 (1988); Nucl. Phys. B328, 1 (1989).

[2] T. Gehrmann and W. J. Stirling, Phys. Rev. D53, 6100 (1996).

[3] G. Altarelli, R. D. Ball, S. Forte, and G. Ridolfi, Nucl. Phys. B496, 337 (1997); Acta Phys. Pol. B29, 1145 (1998).

[4] L. E. Gordon, M. Goshtasbpour, and G. P. Ramsey, Phys. Rev. D58, 094017 (1998).

[5] Spin Muon Collaboration (SMC), B. Adeva et al., Phys. Rev. D58, 112002 (1998).

[6] Asymmetry Analysis Collaboration (AAC), Y. Goto et al., Phys. Rev. D62, 034017 (2000).

[7] D. K. Ghosh, S. Gupta, and D. Indumathi, Phys. Rev. D62, 094012 (2000).

[8] D. de Florian et. al., Phys. Rev. D51, 37 (1995); D57, 5803 (1998); D62, 094025 (2000).

[9] M. Glück, E. Reya, M. Stratmann, and W. Vogelsang, Phys. Rev. D53, 4775 (1996); D63, 094005 (2001).

[10] J. Bartelski and S. Tatur, Z. Phys. C71, 595 (1996); C75, 477 (1997); Phys. Rev. D65, 034002 (2002).

[11] C. Bourrely et. al., Prog. Theor. Phys. 99, 1017 (1998); Eur. Phys. J. C23, 487 (2002).

[12] E. Leader, A. V. Sidorov, and D. B. Stamenov, Phys. Rev. D58, 114028 (1998); Phys. Lett. B462, 189 (1999); Eur. Phys. J C23, 479 (2002).

[13] J. Blümlein and H. Böttcher, Nucl. Phys. B636, 225 (2002).

[14] Spin Muon Collaboration (SMC), B. Adeva et al., Phys. Rev. D58, 112001 (1998).

[15] HERMES collaboration, K. Ackerstaff et al., Phys. Lett. B404, 383 (1997); A. Airapetian et al., Phys. Lett. B442, 484 (1998).

[16] SLAC-E130 collaboration, G. Baum et al., Phys. Rev. Lett. 51, 1135 (1983); E142 collaboration, P. L. Anthony et al., Phys. Rev. D54, 6620 (1996); E143 collaboration, K. Abe et al., Phys. Rev. D58, 112003 (1998); E154 collaboration, K. Abe et al., Phys. Rev. Lett. 79, 26 (1997); E155 collaboration, P. L. Anthony et al., Phys. Lett.
B463, 339 (1999).

[17] SLAC-E155 collaboration, P. L. Anthony et al., Phys. Lett. B493, 19 (2000).

[18] W. T. Giele, S. A. Keller, and D. A. Kosower, hep-ph/0104052 (unpublished).

[19] W. T. Giele and S. Keller, Phys. Rev. D58, 094023 (1998).

[20] D. Stump et al., Phys. Rev. D65, 014012 (2002).

[21] J. Pumplin et al., Phys. Rev. D65, 014013 (2002).

[22] S. I. Alekhin, Phys. Rev. D63, 094022 (2001).

[23] M. Botje, Eur. Phys. J. C14, 285 (2000); J. Phys. G 28, 779 (2002).

[24] CTEQ collaboration, J. Pumplin et al., JHEP 0207, 012 (2002).

[25] ZEUS collaboration, S. Chekanov et al., Phys. Rev. D67, 012007 (2003).

[26] A. D. Martin, R. G. Roberts, W. J. Stirling, and R. S. Thorne, Eur. Phys. J. C28, 455 (2003), hep-ph/0308087

[27] D. Stump et al., JHEP 0310, 046 (2003).

[28] L. W. Whitlow, S. Rock, A. Bodek, S. Dasu, and E. M. Riordan, Phys. Lett. B250, 193 (1990); L. W. Whitlow, report SLAC-0357 (1990); K. Abe et al., Phys. Lett. B452, 194 (1999).

[29] M. Hirai, S. Kumano, and M. Miyama, Comput. Phys. Commun. 108, 38 (1998).

[30] F. James, CERN Program Library Long Writeup D506. See http://wwwasdoc.web.cern.ch/wwwasdoc/minuit /minmain.html.

[31] M. Glück, E. Reya, and A. Vogt, Eur. Phys. J. C5, 461 (1998).

[32] C. Bourrely, E. Leader, and O. V. Teryaev, hep-ph/9803238 (unpublished); G. Altarelli, S. Forte, and G. Ridolfi, Nucl. Phys. B534, 277 (1998).

[33] S. Kumano, Phys. Rep. 303, 183 (1998); G. T. Garvey and J.-C. Peng, Prog. Part. Nucl. Phys. 47, 203 (2001).

[34] T. Morii and T. Yamanishi, Phys. Rev. D61, 057501 (2000); D62, 059901 (2000); M. Stratmann and W. Vogelsang, Phys. Rev. D64, 114007 (2001).

[35] HERMES collaboration, A. Airapetian et al., hep-ex/0307064 
[36] G. Bunce, N. Saito, J. Soffer, and W. Vogelsang, Ann. Rev. Nucl. Part. Sci. 50, 525 (2000).

[37] Jefferson Lab Hall A collaboration, X. Zheng et al., nucl-ex/0308011

[38] http://spin.riken.bnl.gov/aac/ 